\title{
Prevalence and incidence of osteoporotic fractures in patients on long-term glucocorticoid treatment for rheumatic diseases: the Glucocorticoid Induced OsTeoporosis TOol (GIOTTO) study
}

\author{
M. Rossini, O. Viapiana, M. Vitiello, N. Malavolta, G. La Montagna, \\ S. Maddali Bongi, O. Di Munno, R. Nuti, C.U. Manzini, C. Ferri, L. Bogliolo, \\ A. Mathieu, F. Cantatore, A. Del Puente, M. Muratore, W. Grassi, B. Frediani, \\ G. Saviola, P. Delvino, L. Mirone, G. Ferraccioli, G. Tripi, I. Piazza, D. Gatti \\ Osteoporosis and Metabolic Skeletal Diseases Study Group \\ of Italian Society of Rheumatology (SIR)
}

\begin{abstract}
SUMMARY
Osteoporosis and fractures are common and invalidating consequences of chronic glucorticoid (GC) treatment. Reliable information regarding the epidemiology of GC induced osteoporosis (GIOP) comes exclusively from the placebo group of randomized clinical trials while observational studies are generally lacking data on the real prevalence of vertebral fractures, GC dosage and primary diagnosis. The objective of this study was to evaluate the prevalence and incidence of osteoporotic fractures and to identify their major determinants (primary disease, GC dosage, bone mineral density, risk factors, specific treatment for GIOP) in a large cohort of consecutive patients aged $>21$ years, on chronic treatment with GC ( $\geq 5 \mathrm{mg}$ prednisone - PN - equivalent) and attending rheumatology centers located all over Italy. Glucocorticoid Induced OsTeoporosis TOol (GIOTTO) is a national multicenter cross-sectional and longitudinal observational study. 553 patients suffering from Rheumatoid Arthritis (RA), Polymyalgia Rheumatica (PMR) and Connective Tissue Diseases (CTDs) and in chronic treatment with GCs were enrolled.

Osteoporotic BMD values (T score <-2.5) were observed in 28\%, 38\% and 35\% of patients with CTDs, PMR or RA at the lumbar spine, and in $18 \%, 29 \%$ and $26 \%$ at the femoral neck, respectively. Before GC treatment, prevalent clinical fractures were reported by $12 \%, 37 \%$ and $17 \%$ of patients with CTDs, PMR, or RA, respectively. New clinical fragility fractures during GC treatment were reported by $12 \%, 10 \%$ and $23 \%$ of CTDs, PMR and RA patients, respectively. Vertebral fractures were the prevailing type of fragility fracture. More than $30 \%$ of patients had recurrence of fracture. An average of $80 \%$ of patients were in supplementation with calcium and/or vitamin D during treatment with GCs. Respectively, $64 \%, 80 \%$, and $72 \%$ of the CTDs, PMR and RA patients were on pharmacological treatment for GIOP, almost exclusively with bisphosphonates. The GIOTTO study might provide relevant contributions to clinical practice, in particular by highlighting and quantifying in real life the prevalence of GIOP and relative fractures, the frequency of the main risk factors, and the currently sub-optimal prevention. Moreover, these results emphasize the importance of the underlying rheumatic disease on the risk of GIOP associated fractures.
\end{abstract}

Key words: Glucocorticoids; rheumatic diseases; osteoporosis; fractures.

Reumatismo, 2017; 69 (1): 30-39

\section{INTRODUCTION}

Corresponding autho Maurizio Rossini Rheumatology Unit, University of Verona Policlinico Borgo Roma Piazzale Scuro, 10 - 37134 Verona, Italy E-mail: maurizio.rossini@univr.it lucocorticoid therapy (GCs) is a frequently used treatment for rheumatic diseases, such as Rheumatoid Arthritis (RA), Polymyalgia Rheumatica (PMR), and Connective Tissue Diseases (CTDs) such as Systemic Lupus Erythematosus (SLE) (1-3).

In RA the use of low-dose GCs has a welldefined effect on disease activity (4). Different studies about early RA have demonstrat- 
ed that the treatment with disease-modifying anti-rheumatic drugs (DMARDs) in association with GCs may induce high and persistent remission rates (5).

This disease-modifying effect of GCs is supported by different studies (5-12). In addition, the results also showed that GCs have an action in retarding the progression of erosive joint damage in early RA (prior to any joint damage) and a control of disease activity (5-12). The CAMERA II study, in which patients with RA were treated with $10 \mathrm{mg} /$ day of prednisone, has demonstrated that the co-treatment with GCs has a role in the treat-to-target and tight control strategy (13). Many reviews concluded that a low dose of GCs $(<7.5 \mathrm{mg} /$ day $)$ can represent a bridging-therapy while waiting for DMARDs to begin to have effect $(8,13)$. This conclusion was recommended by EULAR in 2010 and then in a 2014 update (1416). The 2012 ACR recommendations update concerned only the use of conventional DMARDs and biological therapies, while it did not recommend the use of GCs (17). In RA the combination therapy with GCs (at a stable low dose of $7.5 \mathrm{mg} /$ day) and traditional DMARDs frequently leads to a suppression of inflammation, and allows a lasting clinical remission (5).

GCs represent the gold standard treatment in PMR, reducing symptoms and suppressing inflammation within a few weeks. GCs act as inhibitors of the circadian release of pro-inflammatory cytokines (such as IL-6), reducing the duration of morning stiffness (2). Although a universal regimen of daily doses of GCs in PMR is not accepted, the EULAR/ACR 2015 recommendations for the management of PMR suggest an initial GCs administration dose within a range of 12.5-25 mg prednisone ( $\mathrm{PN})$, and then of $10 \mathrm{mg}$ for 4-8 weeks before being tapered by $1 \mathrm{mg}$ every $4-8$ weeks (18). In addition, the experts panel discourages conditionally the use of initial doses of $\leq 7.5 \mathrm{mg} /$ day or $>30 \mathrm{mg} /$ day. At the same time, the panel recommends considering the early introduction of methotrexate (MTX) in addition to GCs, particularly in patients at a high risk of relapse. MTX may also be considered during follow-up of patients with a re- lapse, without significant response to GCs or experiencing GC-related adverse events (18). GCs are extensively used also for the treatment of CTDs and their complications or flares.

The use of GCs is associated with the occurrence of side effects, including hypertension, diabetes mellitus, and cataract (19, $20)$. One of the main complications of longterm GCs use is an important alteration of bone metabolism. Bone loss associated with GCs generally involves trabecular and cortical bone, with increased bone resorption but mainly decreased bone formation (21). Bone loss is likely to proceed more rapidly and involves firstly the trabecular compartment because trabecular bone has more available surface upon which the cycle of resorption and formation occurs. Rapid bone loss is marked mostly on endocortical surfaces (22). Doses of GCs $>5 \mathrm{mg}$ $\mathrm{PN}$ equivalent per day are associated with bone loss due to reduced bone formation. Doses of $<5 \mathrm{mg}$ per day may be skeletal sparing, but it is uncertain whether doses $<5 \mathrm{mg}$ are efficacious (23).

Fracture risk is positively related to daily dose and increases during the first 6 months of therapy (24). The relative risk of fractures for patients in GCs therapy is higher for forearm, hip and vertebral sites (24). Some confounding factors such as activity of the disease, age of the patient, sex, baseline BMD, previous fracture history, may influence the rate of bone loss (25). The effects of GCs on fracture risk as well as being dose-related, also depend on the duration of the therapy (26).

Reliable information regarding the epidemiology of GC induced osteoporosis (GIOP) comes exclusively from the placebo group of randomized clinical trials while observational studies are generally lacking data on the real prevalence of vertebral fractures, GC dosage and primary diagnosis.

The main objective of this multicenter study was to evaluate, with a transversal and longitudinal approach, in real life, the prevalence and incidence of osteoporotic fractures and to identify their major determinants in a sample of patients with 
different rheumatic diseases in chronic treatment with GCs. Secondary objectives were to quantify the prevalence of the major risk factors for osteoporotic fracture: age, underlying disease, sex, bone mineral density (BMD), dietary intake of calcium, specific anti-osteoporotic therapy, estimate of vitamin $\mathrm{D}$, physical activity, smoking, alcohol, family history, comorbidity and falls.

\section{MATERIALS AND METHODS}

This is a national multicenter cross-sectional and longitudinal observational study (The Glucocorticoid Induced OsTeoporosis TOol, GIOTTO Study). 553 patients suffering from RA, PMR and CTDs and in chronic treatment with GCs were enrolled in the Italian rheumatologic centers of Verona, Vercelli, Mantova, Pavia, Modena, Bologna, Florence, Pisa, Siena, Ancona, Rome, Naples, Foggia, Lecce, Reggio Calabria, Catania, and Cagliari.

The study was purely observational so no additional treatments or tests other than those normally applied to these patients, were provided or requested.

Each center enrolled patients arriving consecutively in their outpatients clinics and within the following inclusion criteria:

- for the cross-sectional phase: women or men age $>21$ years and receiving GCs for at least one year at a dose of at least $5 \mathrm{mg} /$ day of PN or equivalent

- for the longitudinal phase: patients in therapy with GCs (at least $5 \mathrm{mg} /$ day) for less than three months and in which a therapy with GCs was expected for at least one year at a dosage of at least $5 \mathrm{mg} /$ day of PN or equivalent.

All subjects with at least one of the following characteristics were excluded from the study: a diagnosis of metabolic bone diseases or other forms of secondary osteoporosis (uncontrolled hyperthyroidism, M. Cushing, malabsorption syndrome, primary hyperparathyroidism), severe renal insufficiency (serum creatinine $>2 \mathrm{mg} / \mathrm{dl}$ ), severe liver failure. Patients on aromatase inhibitors, androgen deprivation therapy and heparin were also excluded.

Additional diagnostic tests (instrumental or biochemical) deemed appropriate by the investigator for the clinical practice were provided. The prevalence and incidence of fractures were referred to so-called clinical fractures (but also to any vertebral fractures detected incidentally by radiographic investigation of the spine).

The data collection cards (CRF) included information about medical history, clinical, diagnostic and therapeutic data, in particular: demographics; physiological and pathological history; assessment of the state of the rheumatic disease and concomitant Health Assessment Questionnaire; previous drug therapy; previous and ongoing GC therapy; specific medical history for osteoporosis (date of first diagnosis; date of first fragility fracture; previous vertebral and non-vertebral fractures before the start of GC treatment; new fractures on GC treatment; recurrence of vertebral fractures; risk factors for osteoporosis or fracture); outcome of laboratory examinations; previous/ongoing drug therapy for osteoporosis; assessment of the quality of life (EQ-5D); simplified questionnaire on calcium and vitamin D intake and physical activity.

The study was approved by the Ethics Committee and by the local coordinator. All patients provided written informed consent.

Descriptive statistics analysis included: sample size, mean, standard deviation, median and range for continuous vari-

Table I - Features of Patients depending on the Rheumatic Disease (Mean \pm SD).

\begin{tabular}{|l|c|c|c|c|c|c|}
\hline Rheumatic diseases & No. & F/M & Age (yrs) & BMI (kg/m) & Cumulative dose (g) & Length of therapy (mo) \\
\hline AR & 183 & $157 / 26$ & $64 \pm 13^{*}$ & $25 \pm 5$ & $20.3 \pm 22.9$ & $108 \pm 91$ \\
\hline CTDs & 249 & $206 / 43$ & $57 \pm 16^{*}$ & $25 \pm 4$ & $23.9 \pm 42.9$ & $79 \pm 84^{*}$ \\
\hline PMR & 91 & $71 / 20$ & $74 \pm 6^{*}$ & $26 \pm 5^{*}$ & $6.3 \pm 10.1^{*}$ & $29 \pm 53^{*}$ \\
\hline
\end{tabular}

${ }^{*} \mathrm{p}<0.05$ between groups. 
ables; absolute and relative frequency distributions for categorical variables.

\section{RESULTS}

A total of 553 patients with RA, CTDs (mainly SLE $=93 \%$ ) or PMR in chronic treatment with GCs were recruited. Table I shows the main clinical features of the patients depending on the disease.

The prevalence of the main risk factors for osteoporosis and fracture, according to the disease that requested GCs chronic therapy, is shown in Table II.

The prevalence of osteoporosis according to DXA (T-score $<-2.5$ ) was at the spine in $28 \%, 38 \%$ and $35 \%$ of patients with CTDs, PMR or RA; at the femur, on the other hand, it was respectively 18\%, 29\% and $26 \%$ (Figure 1).

A prevalence of previous clinical fractures was found in $12 \%, 37 \%$ and $17 \%$ of patients suffering respectively from CTDs, PMR, or RA before any treatment with GC (Figure 2A).

During treatment with GC new clinical fragility fractures were reported in $12 \%, 10 \%$ and $23 \%$ of patients suffering respectively from CTDs, PMR and RA (Figure 2B).

The prevailing type of fragility fracture was vertebral, in particular multiple vertebral fractures in patients suffering from PMR (Figure 3A).

More than $30 \%$ of patients had recurrence of vertebral fracture. A second relapse of a vertebral fracture was found in

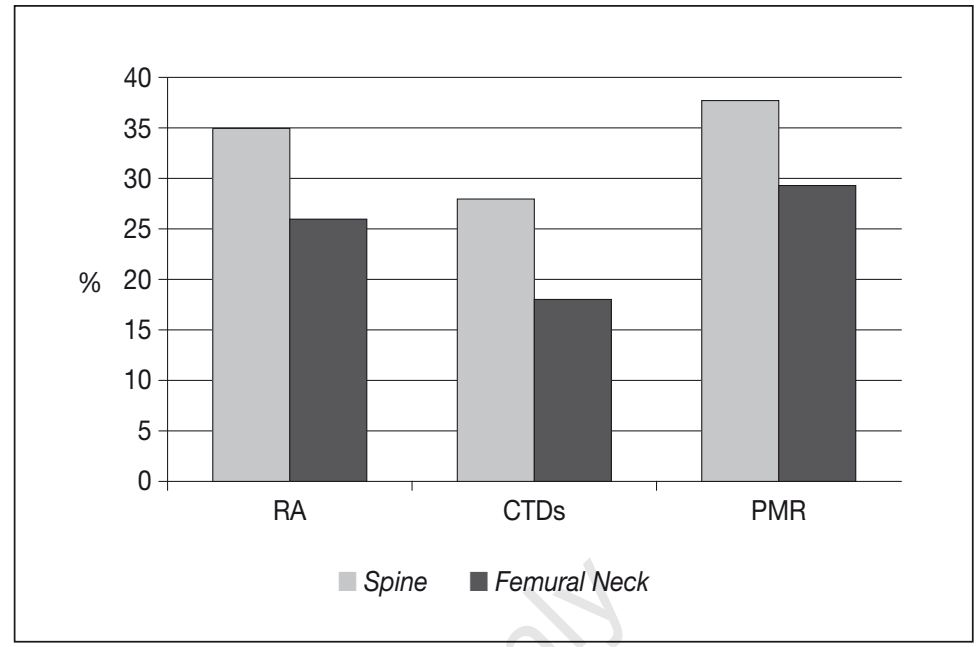

Figure 1 - Prevalence of osteoporosis at spine or femoral neck depending on the Rheumatic Disease.

Table II - Prevalence of risk factors for osteoporosis or fracture, according to the Rheumatic Disease.

\begin{tabular}{|l|c|c|c|}
\hline Risk factors & RA & CTDs & PMR \\
\hline Family history of fractures & $15 \%$ & $23 \%$ & $13 \%$ \\
\hline Alcohol habits & $20 \%$ & $19 \%$ & $34 \%$ \\
\hline Smoking habits (currently/previous) & $19 \% / 23 \%$ & $19 \% / 17 \%$ & $9 \% / 26 \%$ \\
\hline Falls during last 6 months & $20 \%$ & $15 \%$ & $19 \%$ \\
\hline No physical activity & $42 \%$ & $42 \%$ & $39 \%$ \\
\hline Sunlight exposure <1/week & $37 \%$ & $35 \%$ & $18 \%$ \\
\hline No seaside holiday & $66 \%$ & $63 \%$ & $60 \%$ \\
\hline Low calcium intake & $30 \%$ & $23 \%$ & $34 \%$ \\
\hline Comorbidities & $78 \%$ & $76 \%$ & $98 \%$ \\
\hline VAS-EQ-5D<50\%* & $23 \%$ & $18 \%$ & $20 \%$ \\
\hline
\end{tabular}

*Visual analogue scale for recording European Quality of Life-5 Dimensions.
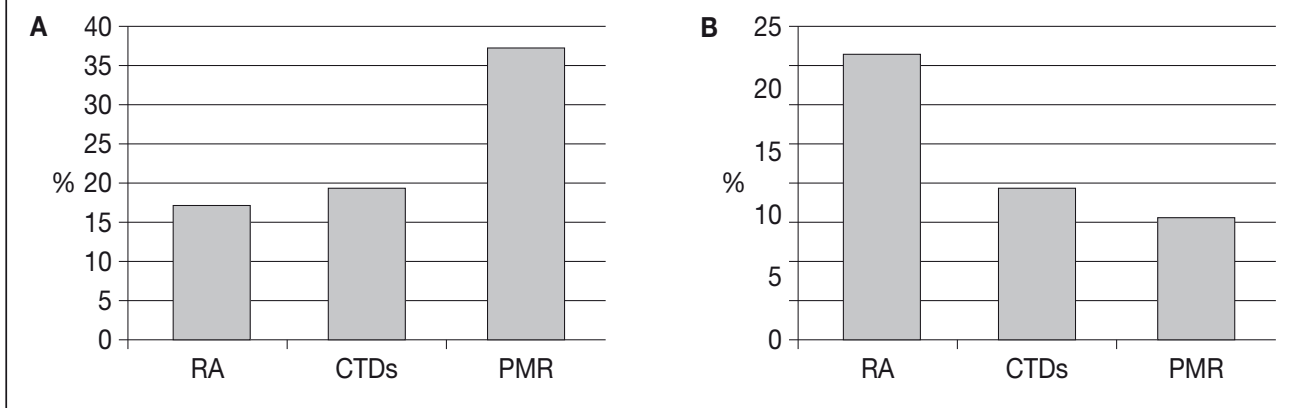

Figure 2 - A) Prevalence of patients with fractures pre-GC treatment, according to the Rheumatic Diseases; B) Prevalence of patients with new clinical fragility fractures in the different Rheumatic Diseases during GC treatment. 


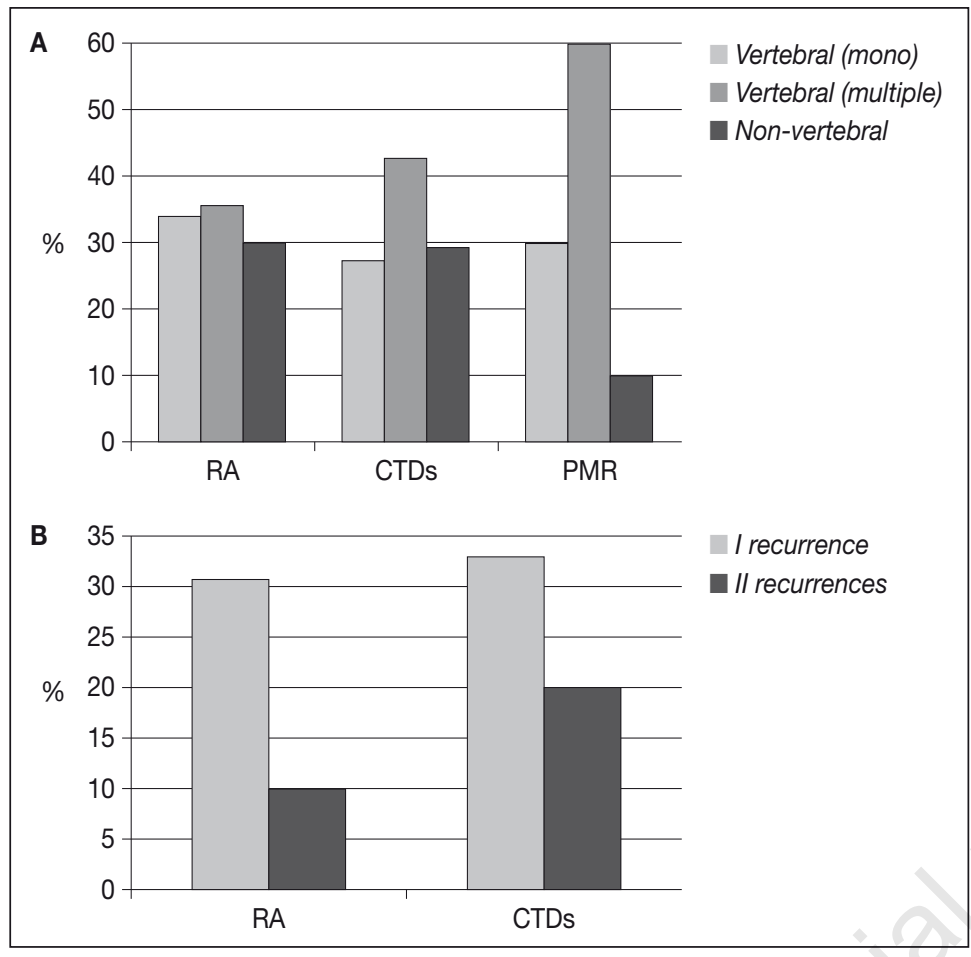

Figure 3 - A) Distribution of different types of fractures, in the different diseases, during GC treatment; B) Prevalence of RA or CTDs patients with recurrence of fractures during $\mathrm{GC}$ treatment.

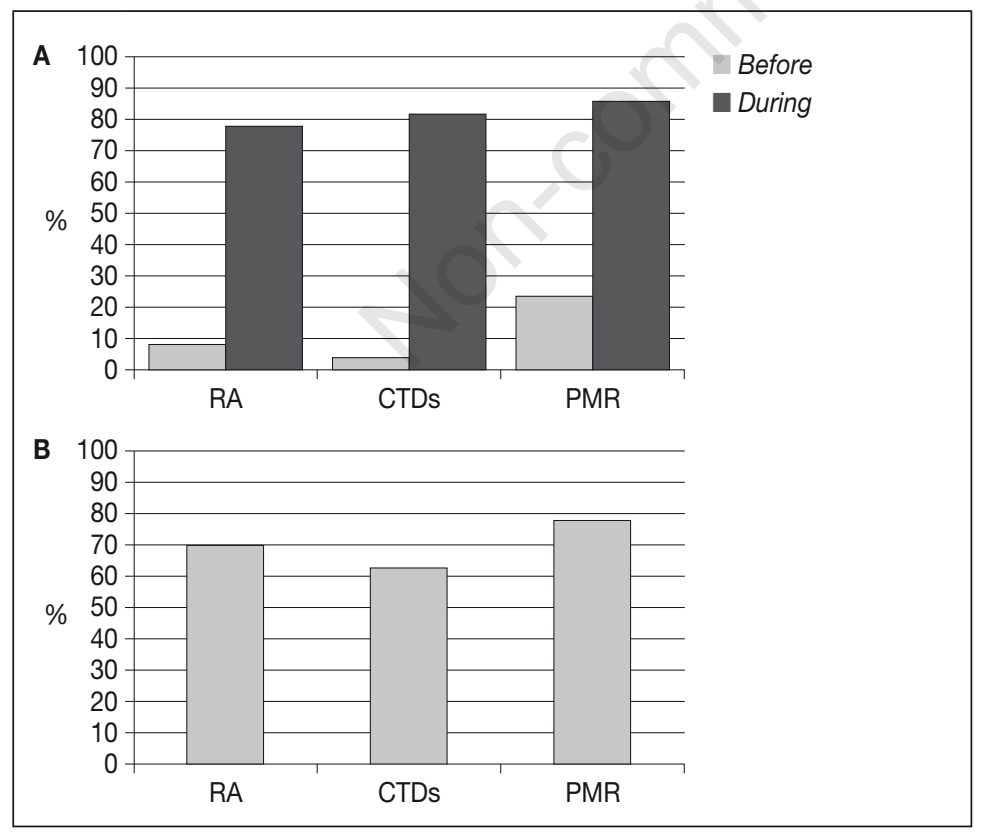

Figure 4 - A) Prevalence of patients with calcium and/or vitamin D supplementation, before or during GC treatment, according to the Rheumatic Disease; B) Prevalence of patients on prevention or treatment of osteoporosis with specific drugs, depending on the Rheumatic Disease.
$20 \%$ of patients with CTDs and $10 \%$ of those with RA (Figure 3B).

In addition, considering only calcium and/ or vitamin $\mathrm{D}$, an average of $80 \%$ of patients were in supplementation during treatment with GCs; only few of them were already on treatment before the beginning of the GCs treatment (Figure 4A).

$64 \%$ of patients with CTDs, $80 \%$ of patients with PMR and $72 \%$ of patients with RA, were treated with specific drugs for the prevention or treatment of GIOP, and in particular with anti-resorptive drugs (alendronate or risedronate) (Figure 4B).

The patients included in the prospective longitudinal phase of the study, which met the inclusion and exclusion criteria, were 83. Their assessment did not show any significant results in addition to those shown by the retrospective longitudinal analysis of 553 cases.

\section{DISCUSSION AND CONCLUSIONS}

The main aim of this multicenter study was to evaluate the prevalence and the incidence of osteoporotic fractures in a cohort of patients with different rheumatic diseases and in therapy with chronic GCs.

In this study we have observed that in RA patients in treatment with GCs, the prevalence of osteoporosis according to DXA, in terms of T-score $<-2.5$, was of $35 \%$ at the spine, while it was $26 \%$ at the femur. In previous studies the frequency of generalized osteoporosis in the global population of patients with RA, with or without GC treatment, ranged from 12.3 to $38.9 \%$ in the lumbar spine and from 6.3 to $36.3 \%$ in the hip (27-29). The frequencies of osteoporosis in the subgroups of RA patients according to current prednisolone use were $26.6 \%$ and $24.7 \%$ at the spine and femoral neck, respectively (27). The KORONA database (including $77.7 \%$ GCs users) showed that the frequency of osteoporosis was $46.8 \%$ (30), a higher percentage in comparison to some other studies, which reported a prevalence of $22-24 \%$, but with a lower prevalence of GC use $(31,32)$. In a large Italian multicenter cross-sectional study that in- 
cluded 925 female RA patients, the authors reported that the frequency of osteoporosis in the whole sample was $28.8 \%$ at the lumbar spine and $36.2 \%$ at the femoral neck. GC use was associated with significantly lower lumbar and femoral BMD (28).

In our study the prevalence of osteoporosis in patients with CTDs (mainly SLE) was of $28 \%$ at the spine and $18 \%$ at the hip. Recently, Carli et al observed that more than $50 \%$ of SLE patients chronically treated with GCs had a reduced BMD and 28\% had osteoporosis (33). In previous studies the prevalence of osteoporosis in GC treated patients with SLE ranged from 1.4 to $68 \%(34,35)$. Two recent studies have demonstrated that bone loss occurs mainly in SLE patients treated with at least $7.5 \mathrm{mg} /$ day prednisone, while treatment with lower doses of prednisone is not associated with bone loss $(36,37)$.

The frequency of osteoporosis in patients with PMR in previous studies ranges between 14.9 and $85 \%$. In our experience, in these elderly patients osteoporosis was generally found in $38 \%$ at the spine and $29 \%$ at the hip.

Risk of fracture in patients who received long-term GC is about 33-50\%, depending on daily and cumulative dose (38). This variability can be explained by the use of different age groups in the study populations, the menopausal status of the participants, and the use of GCs. In addition, we observed that the prevalence of previous clinical fractures before any GCs treatment was $12 \%$ in patients with CTDs, $37 \%$ in patients with PMR and $17 \%$ in patients with RA. This finding may be determined by the role of an underlying inflammation, with the exception of PMR where other factors such as an older age or a higher prevalence of comorbidities could play a role. In the general population, even small elevations of $\mathrm{C}$ reactive protein within the normal range increase non-traumatic fracture risk (39). In some studies, the levels of inflammatory markers and cytokines predicted bone loss and were predictive for fractures $(40,41)$. There is a strong biological rationale for these observations. Osteoclastogenesis is under the control of
RANK-ligand, which is mainly produced by osteocytes in normal bone remodelling, but also by lymphocytes and fibroblasts in other conditions such as inflammation (42). Osteoclastogenesis can be enhanced by many cytokines: interleukin (IL) IL-6, IL-23, Tumor necrosis factor $\alpha$ (TNF- $\alpha$ ). TNF- $\alpha$ transgenic mice are models of osteoporosis with dramatic decrease in bone mass and deterioration of bone microarchitecture. In addition, an over-expression of a Wnt inhibitor, sclerostin, has been observed in these models, with a consequence of inflammation-related decrease in bone formation (43). Moreover, alterations in other Wnt inhibitors, such as DKK1, might be involved in inflammation-related bone loss (44). These findings show that inflammation has a deleterious effect on bone remodelling, inducing increased resorption and decreased formation, before any effect of GCs themselves. Paradoxically, GCs might play a dual role: they induce bone loss and on the other hand they suppress systemic inflammation with a subsequent beneficial effect on bone mass (3).

Autoimmunity, too, might have a role in bone remodelling. For example, in RA the adaptive immune system produces antibodies against rheumatoid factor (RF) and/ or against cyclic citrullinated peptides (antibodies to citrullinated protein antigens or ACPA). The plasma levels of ACPA are an index of disease activity and joint damage (45). A recent study has shown that ACPA can stimulate osteoclast differentiation, and that in ACPA-positive patients with RA there is more frequent evidence of bone loss and thinning of cortical thickness than in APCA-negative patients with RA (46-48). Recently, a significant correlation has been observed between RA related osteoporosis and risk of erosions $(49,50)$. The incidence of new fragility fractures during GCs treatment in our cohort of patients was $12 \%$ in CTDs, $10 \%$ in PMR, and $23 \%$ in RA. The prevailing type of fracture was vertebral, in particular multiple vertebral fractures, especially in patients with PMR. It is known that the risk of fractures is doubled in patients with GCs, and the risk of vertebral fractures is even higher. 
In a study comparing 244,235 oral GCs users versus controls, the relative risk of hip fracture was 1.6, and the relative risk of vertebral fracture was 2.6. Many studies have confirmed these results $(24,51$, 52). The global prevalence of fractures in patients receiving long-term GCs has been reported as $30-50 \%$. In 551 patients receiving long-term GCs, the prevalence of vertebral fractures was $37 \%$, with $14 \%$ of patients having 2 or more asymptomatic vertebral fractures; $48 \%$ of patients aged $\geq 70$ years and $30 \%$ of those aged $<60$ years had at least one vertebral fracture (53). The increase in fracture risk is immediate, as early as 3 months after the initiation of therapy and it reverses sharply after discontinuation of GCs (24). This can be related to the added effects of GCs on bone remodeling previously unleashed by the inflammation itself, and the dramatic effect on bone strength through induced apoptosis of osteocytes. Data also suggest a rapid increase in the rate of falls after the start of oral GCs (24). In epidemiological studies, the increased risk of fractures is observed even at low doses of prednisone, that is, 2.5-5 mg per day. The appropriate care of patients receiving such low doses is not well defined. There is a dose-dependent increase in fracture incidence. Interestingly, the fracture risk is related to the current daily dose, more than to the cumulative dose (54): this may be related to the difficulty of an accurate calculation of this cumulative dose.

Secondary objectives of this study were to quantify the prevalence of the principal risk factors for osteoporotic fractures. The prevalence of comorbidities was very high, especially in PMR. No physical activity was found in about $40 \%$ of the patients, independently of the rheumatic disease, and the prevalence of falls was high, in particular in RA and PMR (about 20\%). Immobilization due to pain from inflamed joints and impairment of physical activity are known key factors related to low BMD and are common in patients suffering from rheumatic diseases (55). The risk of falls may be higher in RA patients because of lower limb joint disease and muscle weak- ness (due to GCs therapy and disuse) (56). An increased fall risk in $89 \%$ of the RA patients was found (57). Potential interventions to reduce falls in this kind of patient include control of disease activity, rehabilitation or exercise therapy, balance training, occupational therapy, home assessment and treatment of vitamin D deficiency. A study by Bearne et al. suggested that a brief rehabilitation can improve the quadriceps sensorimotor function (58).

In addition, in our patients we found high prevalence of low sunlight exposure and low calcium intake. Vitamin D deficiency is common in patients with rheumatic disease, and associated with disease activity (59-61). We also evaluated the regular intake of calcium and vitamin D supplements before and during therapy with GCs. We observed that only a few patients were on supplementation before starting GCs therapy, while about $80 \%$ of patients were taking calcium or vitamin D supplements during GCs therapy, as recommended (62). In our study, prevention or treatment of GIOP with specific drugs for osteoporosis appear sub-optimal, and generally restricted to anti-resorptive approach with bisphosphonates (63), despite it is well known that inhibition of bone formation and increased apoptosis of osteocytes play a consistent and crucial role in the pathogenesis, while changes in bone resorption during GCs use are variable. Although it has been shown that bisphosphonates reduce vertebral fractures during the first 2 years of GC treatment, there are no data on long-term use, and, it is known that they reduce bone turnover, including bone formation, which is already downwards regulated by GCs. Effectively, the use of the anabolic agent teriparatide was found more successful in reducing vertebral fractures than alendronate (64).

This study has a number of limitations, mainly linked to its cross-sectional design and patient selection bias. Moreover, in order to define severe renal insufficiency, a glomerular filtration rate would have been better than serum creatinine cut off, given the muscle wasting in rheumatic chronic diseases (65). 
In conclusion, the GIOTTO study might provide relevant contributions to clinical practice, in particular by highlighting and quantifying in real life the prevalence of GIOP and relative fractures, the frequency of the main risk factors, and the currently sub-optimal prevention. Moreover, these results emphasize the importance of the underlying rheumatic disease on the risk of GIOP associated fractures.

Acknowledgements: the authors thank Sara Rossini who provided editorial assistance.

Funding: this study was supported by an unlimited and unrestricted grant from Eli Lilly.

Conflict of interest: the authors declare no potential conflict of interest.

\section{REFERENCES}

1. Sokka T, Toloza S, Cutolo M, et al. Women, men, and rheumatoid arthritis: analyses of disease activity, disease characteristics, and treatments in the QUEST-RA study. Arthritis Res Ther. 2009; 11: R7.

2. Hernandez-Rodriguez J, Cid MC, Lopez-Soto A, et al. Treatment of polymyalgia rheumatica: a systematic review. Arch Intern Med. 2009; 169: 1839-50.

3. Bultink IE, Lems WF. Systemic lupus erythematosus and fractures. RMD Open. 2015; 1 : e000069.

4. Caporali R, Todoerti M, Sakellariou G, Montecucco C. Glucocorticoids in rheumatoid arthritis. Drugs. 2013; 73: 31-43.

5. Kirwan JR. The effect of glucocorticoids on joint detruction in rheumatoid arthritis. The Arthritis and Rheumatism Council Low-Dose Glucocorticoid Study Group. N Eng J Med. 1995; 333: 142-6.

6. Boers M, Verhoeven AC, Markusse HM, et al. Randomised comparison of combined step-down prednisolone, methotrexate and sulphasalazine with sulphasalazine alone in early rheumatoid arthritis. Lancet. 1997; 350: 309-18.

7. van Everdingen AA, Jacobs JW, Siewertsz Van Reesema DR, Bijlsma JW. Low-dose prednisone therapy for patients with early active rheumatoid arthritis: clinical efficacy, diseasemodifying properties, and side effects: a randomized, doubleblind, placebocontrolled clinical trial. Ann Intern Med. 2002; 136: 1-12.
8. Wassenberg S, Rau R, Steinfeld P, Zeidler H. Very low-dose prednisolone in early rheumatoid arthritis retards radiographic progression over two years: a multicenter, double-blind, placebocontrolled trial. Arthritis Rheum. 2005; 52: 3371-80.

9. Svensson B, Boonen A, Albertsson K, et al. Low-dose prednisolone in addition to the initial disease-modifying antirheumatic drug in patients with early active rheumatoid arthritis reduces joint destruction and increases the remission rate: a two-year randomized trial. Arthritis Rheum. 2005; 52: 3360-70.

10. Kirwan JR, Bijlsma JW, Boers M, Shea BJ. Effects of glucocorticoids on radiological progression in rheumatoid arthritis. Cochrane Database Syst Rev. 2007; 1: CD006356.

11. Goekoop-Ruiterman YP, de Vries-Bouwstra JK, Allaart CF, et al. Comparison of treatment strategies in early rheumatoid arthritis: a randomized trial. Ann Intern Med. 2007; 146: 406-15.

12. Choy EH, Smith CM, Farewell V, et al. Factorial randomised controlled trial of glucocorticoids and combination disease modifying drugs in early rheumatoid arthritis. Ann Rheum Dis. 2008; 67: 656-63.

13. Jabobs JWG. The CAMERA (Computer- Assisted Management in Early Rheumatoid Arthritis) studies. Clin Exp Rheumatol. 2012; 30: S39-43.

14. Gorter SL, Bijlsma JW, Cutolo M, et al. Current evidence for the management of rheumatoid arthritis with glucocorticoids: a systematic literature review informing the EULAR recommendations for the management of rheumatoid arthritis. Ann Rheum Dis. 2010; 69: 1010-4.

15. Smolen JS, Aletaha D, Bijlsma JW, et al. Treating rheumatoid arthritis to target: recommendations of an international task force. Rheum Dis. 2010; 69: 631-7.

16. Smolen JS, Landewé R, Breedveld FC, et al. EULAR recommendations for the management of rheumatoid arthritis with synthetic and biological disease-modifying antirheumatic drugs: 2013 update. Ann Rheum Dis. 2014; 73: 492-509.

17. Singh JA, Furst DE, Bharat A, et al. 2012 update of the 2008 American College of Rheumatology recommendations for the use of disease-modifying antirheumatic drugs and biologic agents in the treatment of rheumatoid arthritis. Arthritis Care Res (Hoboken). 2012; 64: 625-39.

18. Dejaco C, Singh YP, Perel P, et al. Recommendations for the management of polymyalgia rheumatica: a European League Against Rheumatism/American College of Rheumatology collaborative iniziative. Ann Rheum Dis. 2015; 74: 1799-807.

19. van Tuyl LH, Plass AM, Lems WF, et al. Why 
are Dutch rheumatologists reluctant to use the COBRA treatment strategy in early rheumatoid arthritis? Ann Rheum Dis. 2007; 66: 974-76.

20. van der Goes MC, Jacobs JW, Boers M, et al. Patient and rheumatologist perspectives on glucocorticoids: an exercise to improve the implementation of the European League Against Rheumatism (EULAR) recommendations on the management of systemic glucocorticoid therapy in rheumatic diseases. Ann Rheum Dis. 2010; 69: 1015-21.

21. Pocock NA, Eisman JA, Dunstan CR, et al. Recovery from steroid-induced osteoporosis. Ann Intern Med. 1987; 107: 319-23.

22. Henneicke H, Herrmann M, Kalak R, et al. Corticosterone selectively targets endo-cortical surfaces by an osteoblast-dependent mechanism. Bone. 2011; 49: 733-42.

23. Pearce G, Ryan PFJ, Delmas PD, et al. The deleterious effects of low-dose corticosteroids on bone density in patients with polymyalgia rheumatica. Br J Rheumatol. 1998; 37: 292-9.

24. Van Staa TP, Leufkens HG, Abenhaim L, et al. Use of oral corticosteroids and risk fractures. J Bone Miner Res. 2000; 15: 993-1000.

25. da Silva JA, Jacobs JW, Kirwan JR, et al. Safety of low dose glucocorticoid treatment in rheumatoid arthritis: published evidence and prospective trial data. Ann Rheum Dis. 2006; 65: 285-93.

26. Vestergaard P, Olsen ML, Paaske JS, et al. Corticosteroid use and risk of hip fracture: a population-based case-control study in Denmark. J Intern Med. 2003; 254: 486-93.

27. Haugeberg G, Uhlig T, Falch JA, et al. Bone mineral density and frequency of oteoporosis in female patients with rheumatoid arthritis: results from 394 patients in the Oslo County Rheumatoid Arthritis register. Arthritis Rheum. 2000; 43: 522-30.

28. Sinigaglia L, Nervetti A, Mela Q, et al. A multicenter cross sectional study on bone mineral density in rheumatoid arthritis. Italian Study Group on Bone Mass in Rheumatoid Arthritis. J Rheumatol. 2000; 27: 2582-9.

29. Guler-Yuksel M, Bijsterbosch J, GoekoopRuiterman YP, et al. Bone mineral density in patients with recently diagnosed, active rheumatoid arthritis. Ann Rheum Dis. 2007; 66: 1508-12.

30. Lee TJH, Sung YK, Choi CB, et al. The frequency of and risk factors for osteoporosis in Korean patients with rheumatoid arthritis. BMC Musculoskelet Disord. 2016; 17: 98.

31. Gonzalez-Lopez L, Gamez-Nava JI, VegaLopez A, et al. Performance of risk indices for identifying low bone mineral density and osteoporosis in Mexican Mestizo women with rheumatoid arthritis. J Rheumatol. 2000; 39: 247-53.

32. Heberlein I, Demary W, Bloching H, et al. Prophylaxis and treatment of osteoporosis in patients with rheumatoid arthritis (ORA study). Z Rheumatol. 2011; 70: 793-8.

33. Carli L, Tani C, Spera V, et al. Risk factors for osteoporosis and fragility fractures in patients with systemic lupus erythematosus. Lupus Sci Med. 2016; 3: e000098.

34. Boyanov M, Robeva R, Popivanov P. Bone mineral density changes in women with systemic lupus erythematosus. Clin Rheumatol. 2003; 22: 318-23.

35. Uaratanawong S, Deesomchoke U, Lertmaharit $S$, Uara-tanawong $S$. Bone mineral density in premenopausal women with systemic lupus erythematosus. J Rheumatol. 2003; 30: 23658.

36. Zhu TY, Griffith JF, Au SK, et al. Bone mineral density change in systemic lupus erythematosus: a 5-year followup study. J Rheumatol. 2014; 41: 1990-7.

37. Jacobs J, Korswagen LA, Schilder AM, et al. Six year followup study of bone mineral density in patients with systemic lupus erythematosus. Osteoporos Int. 2013; 24: 1287-33.

38. Pereira RMR, de Carvalho JF, Canalis E. Glucocorticoid-induced osteoporosis in rheumatic diseases. Clinics 2010; 65: 1197-205.

39. Schett G, Kiechl S, Weger S, et al. Highsensitivity $\mathrm{C}$-reactive protein and risk of non traumatic fractures in the Bruneck study. Arch Intern Med. 2006; 166: 2495-501.

40. Ding C, Parameswaran V, Udayan R, et al. Circulating levels of inflammatory markers predict change in bone mineral density and resorption in older adults: a longitudinal study. J Clinical Endocrinol Metab. 2008; 93: 1952-8.

41. Cauley JA, Danielson ME, Boudreau RM, et al. Inflammatory markers and incident fracture risk in older men and women: the Health Aging and Body Composition Study. J Bone Miner Res. 2007; 22: 1088-95.

42. Charatcharoenwitthaya N, Khosla S, Atkinson EJ, et al. Effect of blockade of TNF- $\triangle$ and interleukine-1 action on bone resorption in early postmenopausal women. J Bone Miner Res. 2007; 22: 724-9.

43. Chen XX, Baum W, Dwyer D, et al. Sclerostin inhibition reverses systemic, periarticular and local bone loss in arthritis. Ann Rheum Dis. 2013; 72: 1732-6.

44. Rossini M, Viapiana O, Adami S, et al. In patients with rheumatoid arthritis, Dickkopf-1 serum levels are correlated with parathyroid hormone, bone erosions and bone mineral density. Clin Exp Rheumatol. 2015; 33: 77-83.

45. Zendman AJ, van Venrooij WJ, Pruijn GJ. Use and significance of anti-CCP autoantibodies in rheumatoid arthritis. Rheumatology (Oxford). 2006; 45: 20-5.

46. Kleyer A, Finzel S, Rech J, et al. Bone loss before the clinical onset of rheumatoid arthritis in subjects with anticitrullinated protein antibodies. Ann Rheum Dis. 2014; 73: 854-60. 
47. Bugatti S, Bogliolo L, Vitolo B, et al. Anticitrullinated protein antibodies and high levels of rheumatoid factor are associated with systemic bone loss in patients with early untreated rheumatoid arthritis. Arthritis Res Ther. 2016; 18: 226.

48. Orsolini G, Caimmi C, Viapiana O, et al. Titerdependent effect of anti-citrullinated protein antibodies on systemic bone mass in rheumatoid arthritis patients. Calcif Tissue Int. 2017 [Epub ahead of print].

49. Rossini M, Bagnato G, Frediani B, et al. Relationship of focal erosions, bone mineral density, and parathyroid hormone in rheumatoid arthritis. J Rheumatol. 2011; 38: 997-1002.

50. Rossini M, Adami G, Viapiana O, et al. Denosumab, cortical bone and bone erosions in rheumatoid arthritis. Ann Rheum Dis. 2016 [Epub ahead of print].

51. van Staa TP, Leufkens HG, Cooper C. The epidemiology of corticosteroid-induced osteoporosis: a meta-analysis. Osteoporos Int. 2002; 13: 777-87.

52. Kanis JA, Johansson H, Oden A, et al. A metaanalysis of prior corticosteroid use and fracture risk. J Bone Miner Res. 2004; 19: 893-9.

53. Angeli A, Guglielmi G, Dovio A, Capelli G et al. High prevalence of asymptomatic vertebral fractures in post-menopausal women receiving chronic glucocorticoid therapy: a cross-sectional outpatient study. Bone. 2006; 39: 253-9.

54. Van Staa TP, Laan RF, Barton IP, et al. Bone density threshold and other predictors of vertebral fracture in patients receiving oral glucocorticoid therapy. Arthritis Rheum. 2003; 48: 3224-9.

55. Lacativa PG, Farias ML. Osteoporosis and Inflammation. Arq Bras Endocrinol Metabol. 2010; 54: 123-32.

56. Fessel KD, Nevitt MC. Correlates of fear of falling and activity limitation among persons with rheumatoid arthritis. Arthritis Care Res. 1997; 10: 222-8.

57. Kaz Kaz H, Johnson D, Kerry S, et al. Fallrelated risk factors and osteoporosis in women with rheumatoid arthritis. Rheumatology (Oxford). 2004; 43: 1267-71.

58. Bearne LM, Scott DL, Hurley MV. Exercise can reverse quadriceps sensimotor dysfunction that is associated with rheumatoid arthritis without exacerbating disease activity. Rheumatology. 2002; 41: 157-66.

59. Rossini M, Gatti D, Viapiana O, et al. Vitamin D and rheumatic diseases. Reumatismo. 2014; 66: 153-70.

60. Rossini M, D’Avola G, Muratore M, et al. Regional differences of vitamin $\mathrm{D}$ deficiency in rheumatoid arthritis patients in Italy. Reumatismo. 2013; 65: 113-20.

61. Rossini M, Maddali Bongi S, La Montagna $\mathrm{G}$, et al. Vitamin D deficiency in rheumatoid arthritis: prevalence, determinants and associations with disease activity and disability. Arthritis Res Ther. 2010; 12: R216.

62. Rossini M, Adami S, Bertoldo F, et al. Guidelines for the diagnosis, prevention and management of osteoporosis. Reumatismo. 2016; 68: 1-39.

63. Rossini M, Orsolini G, Viapiana O, et al. Bisphosphonates in the treatment of glucocorticoid-induced osteoporosis: pros. Endocrine. 2015; 49: 620-7.

64. Saag KG, Zanchetta JR, de Vogelaer JP, et al. Effects of teriparatide versus alendronate for testing glucocorticoid-induced osteoporosis: 36 months results of a randomized, doubleblind controlled trial. Arthritis Rheum. 2009; 60: 3346-55.

65. Webster AC, Nagler EV, Morton RL, Masson P. Chronic Kidney disease. Lancet. 2017; 389: 1238-52. 